\title{
Contaminação de metais em solos da comunidade tradicional Illha Diana, Santos, SP
}

Metal contamination in solls of the tradtional community of Diana Island, Santos, SP

\author{
Leonardo Silveira Takasee ${ }^{1}$, Larissa Felicidade Werkhauser Demarco ${ }^{2}$, Alexandre Musel Barbosa², Marcos Jorgino Blanco ${ }^{2}$ \\ 1 - Mestrando em Ciências (Oceanografia Geológica), Laboratório de Dinâmica Costeira (LDC), Instituto de Oceanografia, Universidade de São Paulo, São Paulo- \\ SP, BRASIL. \\ 2 - Pesquisador, Instituto de Pesquisas Tecnológicas do Estado de São Paulo, Av. Almeida Prado 532, Cidade Universitária, 05508-901, São Paulo, SP, Brasil. \\ E-MALL: LEONARDO.TAKASE@USP.BR, LARISSAF@IPT.BR, MUSELL@@IPT.BR, MARJB@IPT.BR
}

Abstract: Diana Island is a community in Santos, in the state of São Paulo, and is home to approximately 210 people. There are no published studies on local environmental variables, although the community is bordered by the Diana River, under direct influence of the tide entering the estuary. This research sought to analyze the levels of metals accumulated in soil samples collected in the community, using the $\mathrm{X}$-ray fluorescence method as a screening technique. The results showed that the island exhibits soil contamination of chromium ( $\mathrm{Cr}$ ), cobalt ( $\mathrm{Co})$, zinc ( $\mathrm{Zn}$ ), molybdenum (Mo), cadmium (Cd), barium ( $\mathrm{Ba})$, and lead $(\mathrm{Pb})$, in addition to anomalies in concentrations of iron ( $\mathrm{Fe}$ ) and manganese $(\mathrm{Mn})$. Through distribution patterns, it was possible to determine two different types of sources of contamination and their potential cause. This pioneering study of the levels of local metal contamination may serve as a parameter for future contamination studies of Diana Island soil.

Resumo: A llha Diana, inserida no estuário de Santos (SP), abriga aproximadamente 210 pessoas. Sem saneamento básico, 0 bairro é margeado pelo rio Diana, afetado pela maré que adentra 0 estuário, onde são lançados os efluentes gerados pela comunidade. Esta pesquisa objetivou analisar os teores de metais acumulados em amostras de solo do bairro, a partir do uso do método de fluorescência de raio X (XRF) como técnica de varredura. 0 s resultados mostraram que a ilha apresenta valores de contaminação para cromo $(\mathrm{Cr})$, cobalto $(\mathrm{Co})$, zinco $(\mathrm{Zn})$, molibdênio $(\mathrm{Mo})$, cádmio $(\mathrm{Cd})$, bário $(\mathrm{Ba})$ e chumbo $(\mathrm{Pb})$, além de anomalias de concentração para ferro ( $\mathrm{Fe}$ ) e manganês $(\mathrm{Mn})$. Por meio dos padrões de distribuição, foi possível determinar dois tipos distintos de fontes de contaminação e suas causas potenciais. A análise detalhada pode servir de parâmetro para estudos futuros de contaminação no solo da comunidade.
Citation/Citação:Takase, L. S., Demarco, L. F. W., Barbosa, A. M., \& Blanco, M. J. (2020). Contaminação de metais em solos da comunidade tradicional Illha Diana, Santos, SP. Terræ Didatica, 16, 1-7, e020049. doi: 10.20396/ td.v16i0.8661279.

Keywords: Pollution, Heavy metals, Soil, Estuary

Palavras-chave: Poluição, Metais, Solo, Estuário

Manuscript/Manuscrito:

Received/Recebido: 18/09/2020

Revised/Corrigido: 09/11/2020

Accepted/Aceito: 01/12/2020

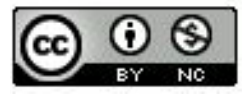

\section{Introdução}

Desde a metade do século 19, processos antrópicos têm causando grandes impactos e modificações ambientais, afetando também ambientes aquáticos (Branchet et al., 2019). No Brasil, estudos para a avaliação da contaminação dos ambientes aquáticos são realizados há muito tempo, principalmente pelo grande impacto que as contaminações podem causar ao abastecimento humano (Dores \& De-Lamonica-Freire, 2001, Veiga, Silva, Veiga, \& Faria, 2006). Neste sentido, as contaminações estão associadas à poluição dos corpos d'agua e a origem da contaminação tem diversas fontes, sendo agravada em áreas portuárias e de atividades industriais (Hortellani, Sarkis, Abessa \& Sousa, 2008).
A Ilha Diana, localizada no estuário de Santos/ SP, está suscetível a diversas fontes de contaminação, seja por despejo de efluentes domésticos, pelo grande número de indústrias nos arredores da região estuarina e pela proximidade da zona portuária. Trata-se de uma comunidade tradicional e uma das poucas colônias de pescadores ainda existentes na região, com acesso ao continente realizado apenas por barcos. Muitos estudos apontam as contaminações do estuário de Santos, SP (Virga, Geraldo \& Santos, 2007, Hortellani et al., 2008), mas pouco se sabe sobre a influência das contaminações sobre a ilha, que sofre intrusão das marés do estuário, e, em algumas ocasiões, fica parcialmente submersa. Nessas ocasiões, o 
sedimento do fundo do estuário que sofre revolvimento é carreado em partes e depositado sobre o solo da ilha.

A varredura por fluorescência de raios-X (XRF) é uma técnica analítica utilizada em estudos de contaminação como ferramenta de varredura, a qual utiliza métodos indiretos e de rápida obtenção de resultados para a determinação de áreas prioritárias aos estudos posteriores, sendo possível realizar quantificações analíticas de baixo custo. Isso permite que seja analisado maior número de amostras, possibilitando maior representatividade espacial dos resultados e melhor determinação da distribuição de potenciais contaminações.

O objetivo deste estudo, portanto, é de avaliar a presença de metais pesados no solo superficial da ilha Diana por meio de técnica de varredura por fluorescência de raios-X (XRF), avaliando a potencial contaminação da área. A seleção dos metais de interesse foi baseada nos elementos apresentados na lista de valores orientadores para solo no Estado de São Paulo (Companhia Ambiental do Estado de São Paulo - Cetesb, 2016), sendo acrescidas as leituras de ferro $(\mathrm{Fe})$, manganês ( $\mathrm{Mn})$ e do cloro $(\mathrm{Cl})$, analisados pelo mesmo método.

\section{Área de Estudo}

A Ilha Diana, inserida na região do estuário de Santos (SP), localiza-se na parte continental do município, na confluência do rio Diana com o canal de Bertioga (Fig. 1). Com área de aproximadamente $37.800 \mathrm{~m}^{2}$ e cerca de 210 moradores, o acesso ao bairro se dá apenas por barco coletivo ou por embarcações de uso pessoal de pequeno porte. Apesar de receber o nome de Ilha, o "status de ilha" ocorre devido à intrusão da maré (Pereira $\&$ Ambrosio, 2018) que inunda a parte em frente ao rio Diana durante o período de enchente. Os estudos já conduzidos sobre o bairro fazem referência, principalmente, a ocupação da ilha, ao modo de vida caiçara local e a expansão dos terminais portuários na região estuarina (Vale, 2011, Stori, Terra \& Abessa, 2012, 2013, Santos

\& Clauzet, 2014, Pereira \& Ambrosio, 2018).

\section{Materiais e Métodos}

Para a seleção dos pontos de amostragem foi avaliada a dinâmica local, sendo identificadas as áreas afetadas pelas condições ambientais (entrada da maré na região continental da ilha e fluxo do rio Diana) e pelas condições antrópicas, como o despejo de esgoto doméstico em regiões da própria comunidade. Os pontos foram distribuídos ao longo do solo do bairro, formando uma malha amostral terrestre, contemplando os locais com influência direta ou indireta de processos ambientais e antrópicos.

De forma a avaliar as potenciais contaminações acumulativas, o solo foi coletado no intervalo entre $15 \mathrm{~cm}$ e $30 \mathrm{~cm}$ de profundidade e sua posição de coleta registrada com GPS manual Garmin. As amostras foram devidamente armazenadas, identificadas e encaminhadas para o Laboratório de Resíduos e Áreas Contaminadas no IPT.

Para as análises de fluorescência de raio-X, as amostras foram secas ao ar para a redução da umidade, destorroadas, passadas em peneira de $20 \mathrm{~cm}$ de diâmetro com malha de $2 \mathrm{~mm}$ (terra fina seca ao 
ar - TFSA) e, posteriormente, homogeneizadas. As leituras foram realizadas em uma alíquota de $100 \mathrm{~g}$ no equipamento Innov-X Alpha Series XRF, com duração de 1 min e em triplicata. Foi realizado o tratamento estatístico dos dados utilizando-se as médias das leituras de cada elemento como valor representativo do elemento para aquela amostra. Já para determinação dos valores anômalos foi utilizada a amplitude interquartil (IQR), os quais foram calculados multiplicando os dados de IQR por 1,5 e obtendo-se, assim, os valores de corte para os elementos identificados.

Os dados foram espacializados no programa ArcGIS, criando a distribuição espacial dos pontos com base nas concentrações quantificadas e interpolando-os por meio do método topo to raster, gerando mapas de distribuição de concentração para cada elemento com um limite espacial determinado.

\section{Resultados e Discussão}

As análises de XRF identificaram concentrações de cromo $(\mathrm{Cr})$, cobalto $(\mathrm{Co})$, cobre $(\mathrm{Cu})$, zinco $(\mathrm{Zn})$, molibdênio $(\mathrm{Mo})$, cádmio $(\mathrm{Cd})$, bário $(\mathrm{Ba})$, chumbo $(\mathrm{Pb})$, os quais estão presentes na lista de valores orientadores para solo da Cetesb (2016). Além disso, também foram identificadas concentrações para ferro $(\mathrm{Fe})$, manganês $(\mathrm{Mn})$ e cloro $(\mathrm{Cl})$. A Tabela 1 apresenta o sumário estatístico para os parâmetros identificados.

Os parâmetros dos elementos $\mathrm{Cr}, \mathrm{Co}, \mathrm{Cu}$, $\mathrm{Zn}, \mathrm{Mo}, \mathrm{Cd}$ e $\mathrm{Pb}$ apresentaram valores mínimos iguais a zero, demonstrando que a presença destes elementos não ocorre de maneira abrangente no solo superficial da ilha. Ao avaliarmos os valores máximos quantificados, entretanto, estes são supe- riores ao valor de corte calculado para a determinação das anomalias, o que sugere a presença de contaminação na área.

Segundo Hawkins (1980), um outlier é um fator que desvia tanto de outros fatores a ponto de gerar suspeitas de que foi gerado por um mecanismo diferente; neste estudo, os outliers demonstram que as concentrações anômalas são decorrentes de processos que não estão ligados aos mecanismos pedogenéticos de formação do solo local. Este comportamento também é observado nos elementos Fe e Mn, tipicamente encontrados em solos brasileiros, mas que aqui apresentaram valores elevados, evidenciando que fatores externos influenciam nas concentrações destes elementos.

O mesmo comportamento também é observado para o $\mathrm{Cl}$, que aqui é utilizado como um indicador natural da influência da subida da maré como processo de deposição de sedimentos sobre o solo da ilha, visto que este é um elemento presente em águas salgadas e salobras. A presença do $\mathrm{Cl}$ e sua distribuição no solo superficial é um indicativo do comportamento e padrão da deposição dos metais sobre influência da maré na ilha Diana, portanto.

A Tabela 2 sumariza os resultados analíticos obtidos pela análise de XRF e os valores anômalos calculados para todas as amostras.

$\mathrm{Cu}, \mathrm{Cd}, \mathrm{Zn}$ e Mo foram identificados em poucas amostras, cuja distribuição se restringe às áreas internas da ilha. Os valores, superiores aos valores de corte, indicam contaminação por substâncias portadoras desses elementos. Não foram identificadas concentrações nas regiões próximas às margens do rio Diana (Fig. 2). O fato de os pontos amostrais estarem próximos a residências corrobora o comportamento da distribuição encontrada: não há correlação espacial entre os valores quantificados,

Tabela 1. Sumário das estatísticas descritivas dos parâmetros analisados no solo de ilha Diana

\begin{tabular}{l|l|l|l|l|l|l|l|l|l|l|l}
\hline Dados/Elementos & $\mathbf{C r}$ & $\mathbf{C o}$ & $\mathbf{C u}$ & $\mathbf{Z n}$ & $\mathbf{M o}$ & $\mathbf{C d}$ & $\mathbf{B a}$ & $\mathbf{P b}$ & $\mathbf{M n}$ & $\mathbf{F e}$ & $\mathbf{C l}$ \\
\hline Média & 12,6 & 7,2 & 2,5 & 27,5 & 8,1 & 1,9 & 117,5 & 14,1 & 143,0 & $6.485,8$ & $1.970,3$ \\
\hline Mediana & 0,0 & 8,0 & 0,0 & 15,0 & 0,0 & 0,0 & 105,3 & 8,7 & 111,0 & $5.657,0$ & 0,0 \\
\hline Mínimo & 0,0 & 0,0 & 0,0 & 0,0 & 0,0 & 0,0 & 44,0 & 0,0 & 32,7 & 471,7 & 0,0 \\
\hline Máximo & 43,0 & 20,0 & 12,7 & 173,0 & 32,5 & 33,0 & 222,0 & 50,3 & 307,7 & $15.220,7$ & $9.340,0$ \\
\hline $\mathbf{1}^{\mathbf{o}}$ Quartil & 0,0 & 0,0 & 0,0 & 6,0 & 0,0 & 0,0 & 69,0 & 5,0 & 48,0 & $2.612,0$ & 0,0 \\
\hline $\mathbf{3}^{\text {o }}$ Quartil & 28,5 & 11,0 & 6,0 & 35,3 & 20,5 & 0,0 & 130,3 & 19,7 & 235,7 & $8.113,7$ & $2.541,3$ \\
\hline Desvio Padrão & 16,5 & 7,2 & 4,2 & 41,5 & 13,2 & 8,0 & 57,0 & 15,0 & 94,3 & $4.296,4$ & $2.946,3$ \\
\hline IQR & 28,5 & 11,0 & 6,0 & 29,3 & 20,5 & 0,0 & 61,3 & 14,7 & 187,7 & $5.501,7$ & $2.541,3$ \\
\hline Outlier & 42,8 & 16,5 & 9,0 & 44,0 & 30,8 & 0,0 & 92,0 & 22,0 & 281,5 & $8.252,5$ & $3.812,0$ \\
\hline
\end{tabular}


Tabela 2. Tabela 02. Resultados analíticos dos elementos analisados no solo de ilha Diana. Os valores anômalos calculados para os parâmetros analisados estão destacados em vermelho.

\begin{tabular}{|c|c|c|c|c|c|c|c|c|c|c|c|}
\hline ID & $\mathrm{Cr}$ & Co & $\mathbf{C u}$ & $\mathrm{Zn}$ & Mo & Cd & $\mathbf{B a}$ & $\mathbf{P b}$ & Mn & $\mathbf{F e}$ & $\mathrm{Cl}$ \\
\hline P02 & 27,00 & 8,00 & 0,00 & 9,33 & 0,00 & 0,00 & 105,33 & 0,00 & 85,00 & 4475,33 & 9340,00 \\
\hline P04 & 40,33 & 20,00 & 0,00 & 15,00 & 0,00 & 0,00 & 222,00 & 7,33 & 257,33 & 15220,67 & 0,00 \\
\hline P06 & 0,00 & 0,00 & 0,00 & 7,67 & 0,00 & 0,00 & 80,33 & 8,67 & 45,33 & 2612,00 & 3370,00 \\
\hline P07 & 43,00 & 14,50 & 0,00 & 17,00 & 0,00 & 0,00 & 217,33 & 10,33 & 236,33 & 13902,67 & 0,00 \\
\hline P08 & 0,00 & 11,00 & 6,50 & 57,67 & 32,50 & 0,00 & 102,00 & 23,33 & 213,33 & 7491,00 & 0,00 \\
\hline P09 & 0,00 & 10,33 & 9,33 & 35,33 & 0,00 & 0,00 & 115,33 & 19,67 & 111,00 & 8113,67 & 2330,67 \\
\hline P10 & 0,00 & 17,00 & 0,00 & 16,67 & 0,00 & 0,00 & 219,67 & 50,33 & 235,67 & 12454,00 & 1737,33 \\
\hline P11 & 17,00 & 0,00 & 6,00 & 42,00 & 26,33 & 33,00 & 66,67 & 15,67 & 145,00 & 4462,67 & 0,00 \\
\hline P12 & 0,00 & 0,00 & 0,00 & 6,00 & 0,00 & 0,00 & 65,00 & 3,00 & 48,00 & 2375,33 & 8594,33 \\
\hline P13 & 0,00 & 18,00 & 0,00 & 51,67 & 0,00 & 0,00 & 93,50 & 6,50 & 251,67 & 7907,33 & 0,00 \\
\hline P14 & 28,50 & 0,00 & 12,67 & 173,00 & 28,00 & 0,00 & 156,33 & 47,67 & 307,67 & 9636,00 & 0,00 \\
\hline P15 & 29,00 & 8,00 & 8,00 & 20,33 & 0,00 & 0,00 & 127,00 & 22,00 & 207,33 & 6092,00 & 0,00 \\
\hline P16 & 30,00 & 9,00 & 0,00 & 7,33 & 0,00 & 0,00 & 124,67 & 13,67 & 97,33 & 5657,00 & 2541,33 \\
\hline P17 & 0,00 & 0,00 & 0,00 & 0,00 & 0,00 & 0,00 & 59,33 & 5,00 & 35,00 & 2522,33 & 3776,67 \\
\hline P18 & 0,00 & 0,00 & 0,00 & 4,33 & 20,50 & 0,00 & 69,00 & 7,00 & 32,67 & 4272,00 & 0,00 \\
\hline P19 & 0,00 & 0,00 & 0,00 & 0,00 & 31,00 & 0,00 & 44,00 & 0,00 & 32,67 & 471,67 & 0,00 \\
\hline P20 & 0,00 & 6,00 & 0,00 & 4,00 & 0,00 & 0,00 & 130,33 & 0,00 & 90,33 & 2593,67 & 1805,00 \\
\hline
\end{tabular}
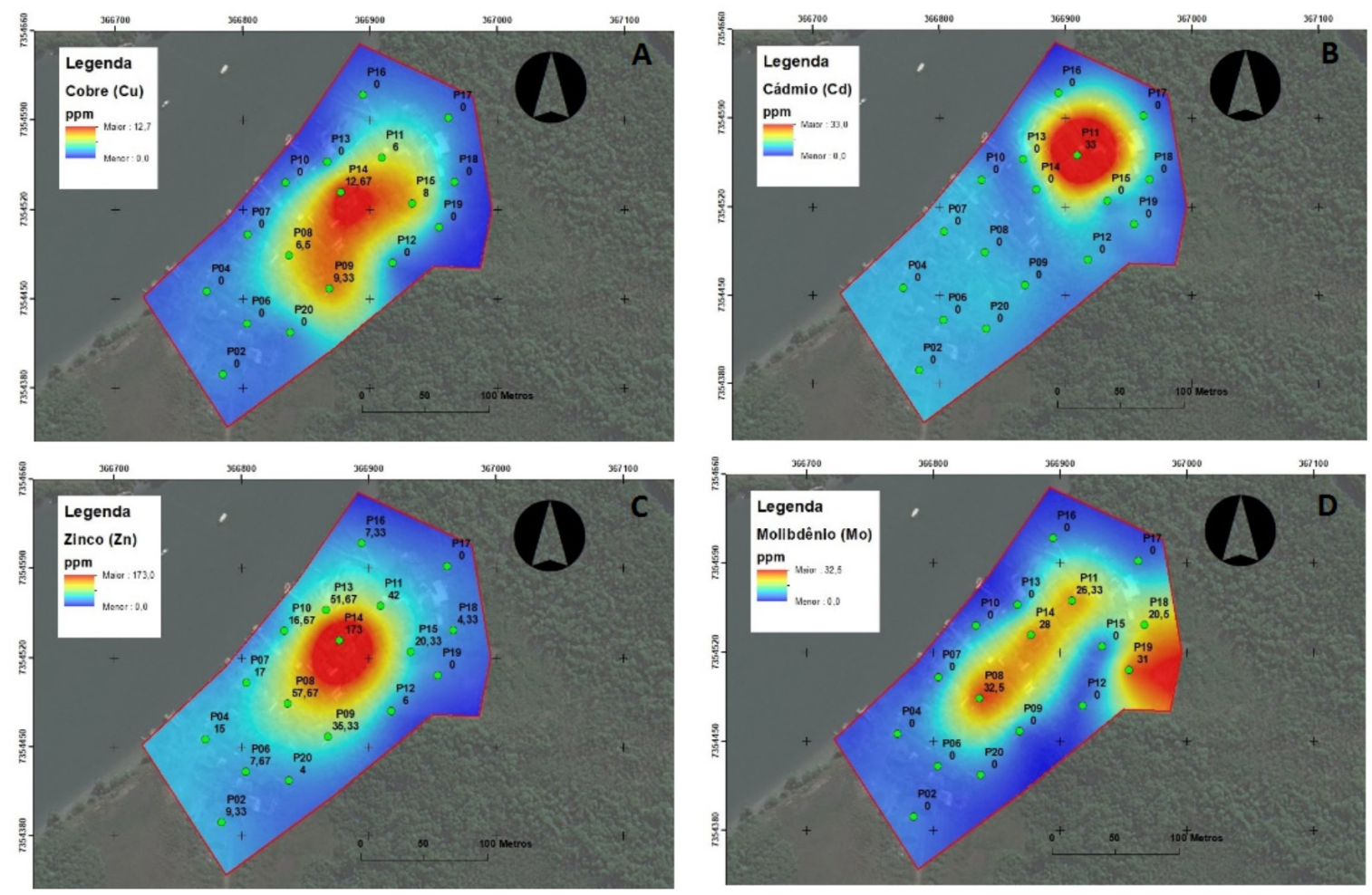

Figura 2. Distribuição das concentrações: A) cobre, B) cádmio, C) zinco e D) molibdênio 
já que a presença dos elementos no solo associa-se às atividades diárias dos moradores.

De forma oposta, o chumbo apresentou maiores concentrações na região próxima ao píer (região vermelha da Figura 3), o que pode estar associado a diversas fontes, uma vez que este elemento faz parte

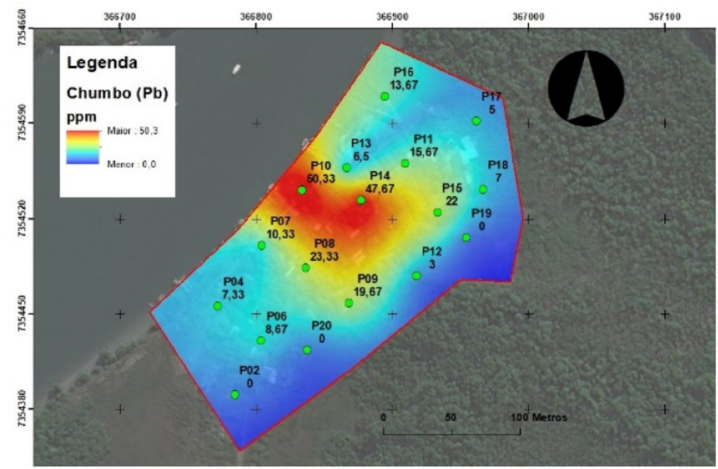

Figura 3. Distribuição das concentrações de chumbo $(\mathrm{Pb})$
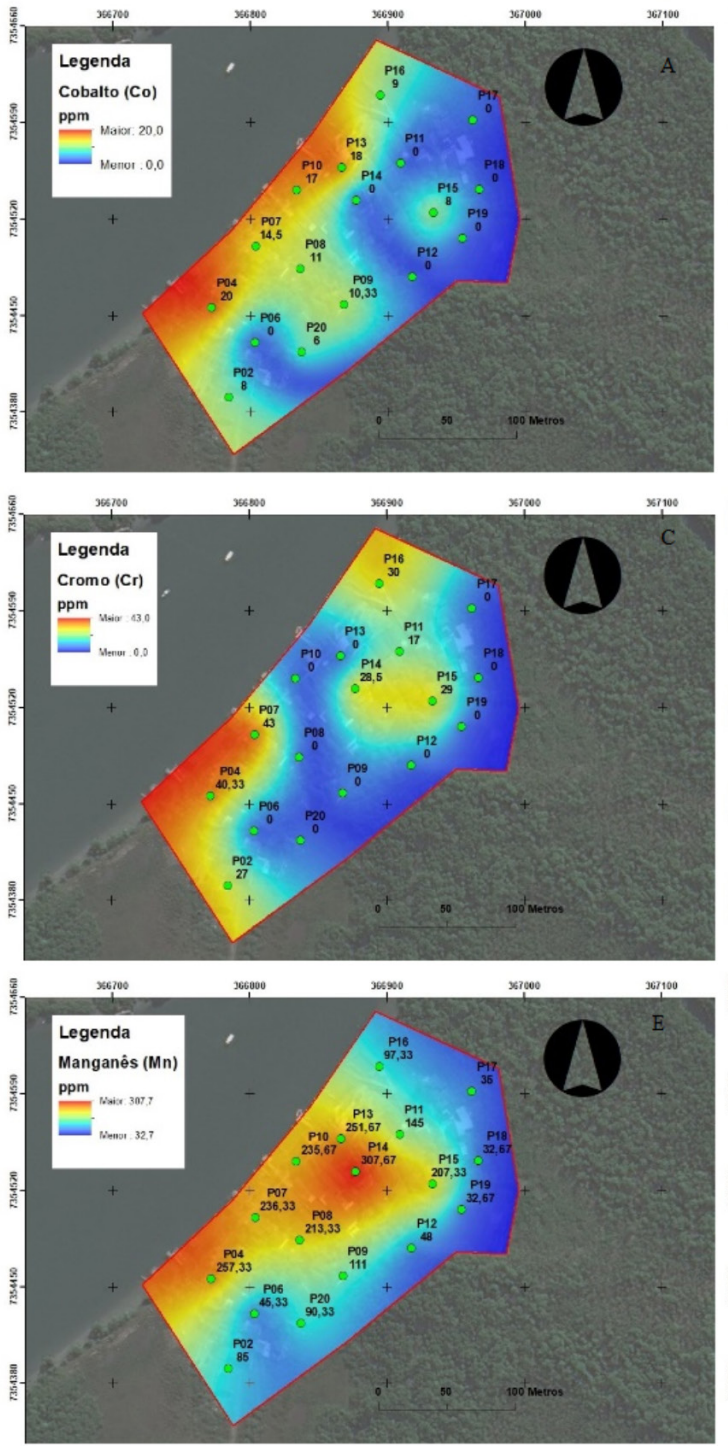

Figura 4. Distribuição das concentrações: A) cobalto; B) bário; C) cromo; D) ferro; E) manganês; F) cloro (C) Terrae Didat.

Campinas, da composição de muitos produtos, sendo o local de amostragem também próximo a redes de esgoto, lançado de forma direta no rio Diana (Demarco, Takase, Blanco \& João, 2019, Demarco et al. 2019), podendo ser uma fonte de contaminação local e, deste modo, a presença anômala de Pb também pode estar associada as atividades antrópicas locais.

Já os elementos cobalto $(\mathrm{Co})$, bário $(\mathrm{Ba})$ e cromo $(\mathrm{Cr})$, presentes na lista de valores orientadores para solo (Cetesb, 2016) e o ferro (Fe), manganês $(\mathrm{Mn})$ e cloro $(\mathrm{Cl})$, analisados como forma de avaliar os elementos de ocorrência natural, apresentaram o mesmo comportamento de distribuição (Fig. 4). Todos esses elementos tiveram valores superiores aos valores de corte para a determinação das anomalias, demonstrando que a sua ocorrência no solo superficial na Ilha Diana supera as concentrações
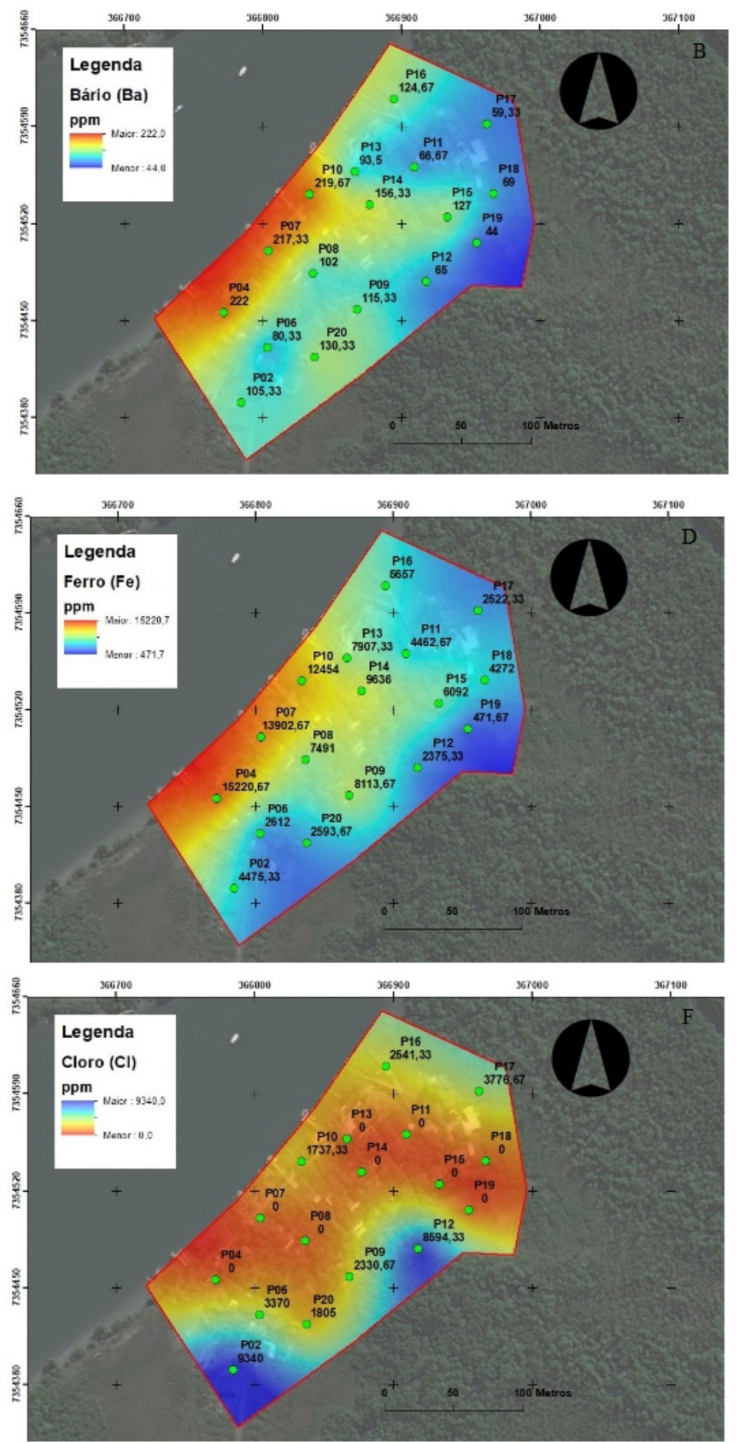
esperadas para valores de ocorrência natural. $\mathrm{O}$ padrão de distribuição das concentrações é de gradiente negativo em direção ao interior da ilha e sua comunidade.

Dado que o $\mathrm{Cl}$ possui influencia direta da deposição dependente das subidas das marés e carreamento de sedimentos é possível afirmar, com base nos comportamentos apresentados pelos outros elementos, que a ocorrência destes valores superiores aos naturais é também decorrente do transporte de sedimentos. Assim, é válido apontar que a acumulação desses metais provavelmente é resultado de uma contaminação externa à área continental da comunidade. Por conseguinte, esses contaminantes podem ser transportados ao longo do sistema estuarino do canal de Santos/SP e serem disponibilizados pela água para o solo do bairro (principalmente durante a maré enchente de sizígia), afetando suas características químicas.

Embora a Cetesb realize estudos sobre a qualidade da água nos rios que compõem o sistema estuarino de Santos/SP, como o rio Piaçaguera ou o rio Cubatão, não há um monitoramento do rio Diana. Além disso, as tabelas com os teores de metais em sedimentos dos rios monitorados pela Cetesb em 2017 (mesmo ano em que foram feitas as coletas deste estudo) não apresentam resultados para a região estuarina de Santos (SP) que, ademais, está próxima ao rio Diana.

\section{Conclusão}

A partir das análises dos teores de metais no solo da comunidade de Ilha Diana e da consequente espacialização dos dados, foi possível observar que existem dois tipos de impactos no solo superficial da ilha: um gerado pelas atividades locais dos moradores, potencialmente associado à falta de saneamento básico, com os efluentes sendo despejados diretamente no rio Diana; e outro, gerado pelo impacto externo à ilha, decorrente da degradação e contaminação do estuário de Santos (SP). Nessa área, devido às ocorrências naturais de variação de maré, depositam-se sedimentos contaminados sobre a ilha. Além disso, a aplicação do XRF como uma ferramenta de varredura se mostrou útil, possibilitando analisar uma gama de metais de interesse de forma rápida e de baixo custo, com posterior processamento e espacialização dos dados. Espera-se, portanto, que esses resultados possam servir como um ponto de partida para estudos posteriores na região.

\section{Agradecimentos}

Os autores agradecem à Fundação de Apoio ao Instituto de Pesquisas Tecnológicas (FIPT), ao Instituto de Pesquisas Tecnológicas do Estado de São Paulo e ao prof. Dr. Ronaldo Torres da Universidade Federal de São Paulo por todo o apoio recebido.

\section{Referências}

Branchet, P., Ariza Castro, N., Fenet, H., Gomez, E., Courant, F., Sebag, D., ... Gonzalez, C. (2019). Anthropic impacts on Sub-Saharan urban water resources through their pharmaceutical contamination (Yaoundé, Center Region, Cameroon). Science of the Total Environment, 660, 886-898. doi: 10.1016/j.scitotenv.2018.12.256

Companhia Ambiental do Estado de São Paulo (Cetesb). 2016. Decisão de diretoria $n^{\circ}$ 256/2016/E, 22 de novembro de 2016. Dispõe sobre a aprovação dos "Valores Orientadores para Solos e Águas Subterrâneas no Estado de São Paulo, 2016" e dá outras providências. São Paulo, Cetesb. URL: https://www.Cetesb.sp.gov.br/wp-content/ uploads/2014/12/DD-256-2016-E-Valores-Orientadores-Dioxinas-e-Furanos-2016-Intranet.pdf.

Demarco, L. F. W., Takase, L. S., Blanco, M. J. \& João, L. de A. Sobrinho. (2019). Abordagem multidisciplinar para indicar as prováveis ameaças à saúde e à qualidade de vida da comunidade caiçara Ilha Diana. Revista Brasileira de Geografia Médica e da Saúde (Hygeia), 15(34), 62-84. doi: 10.14393/Hygeia153449670.

Demarco, L. F. W., Barbosa, A. M., Blanco, M. J., Fonseca, A. F., Takase, L. S., João, L. de A. Sobrinho \& Saldias, F. I. S. (2019). Uso do XRF em amostras de solo da comunidade Ilha Diana - Santos, SP. In F. S. Machado, A. S. de Moura (Orgs), Educação, Meio Ambiente e Território 3 (Vol. 3, Cap. 7, pp. 7178). Ponta Grossa: Atena Editora. doi: 10.22533/ at.ed.4421921027.

Dores, E. F. G. C., De-Lamonica-Freire, E. M. (2001) Contaminação do ambiente aquático por pesticidas. Estudo de caso: águas usadas para consumo humano em primavera do leste, Mato Grosso análise preliminar. Quím. Nova, 24(1), 27-36. doi: 10.1590/S0100-40422001000100007

Hawkins, D. M. (1980). Identification of Outliers. Chapman and Hall, London. 188 p. doi: 10.1007/97894-015-3994-4

Hortellani, M. A., Sarkis, J. E. S, Abessa, D. M. S., Sousa, E. C. P. M. (2008). Avaliação da contaminação por elementos metálicos dos sedimentos do estuário Santos - São Vicente. Quím. Nova, 31(1), 1019. doi: 10.1590/S0100-40422008000100003.

Pereira, C. D. E. F. \& Ambrosio, R. P. (2018). Aspectos urbanísticos da informalidade urbana da Ilha Diana e legislação urbanística: alternativas para garantia do direito à moradia e à cidade. Leopoldianum, 44(123), 135-50. URL: https://periodicos.unisantos.br/leopoldianum/article/view/824/694.

Santos, L. A. F. dos, Clauzet, M. (2014). A influência da expansão portuária na comunidade da Ilha Diana, Santos-SP. UNISANTA BioScience, 3(4), 220-225. 
URL: http://sites.unisanta.br/ppgmec/documentos/ANAIS_TERCEIRO_ENCONTRO_POS. pdf\# page $=\overline{7} 8$.

Stori, F., Terra, N. N., Abessa, D. M. S. (2012). Mecanismos socioecológicos e práticas tradicionais de pesca na comunidade caiçara da Ilha Diana (Santos, Brasil) e suas transformações. Revista de Gestão Costeira Integrada, 12(4), 521-533. URL: http:// www.scielo.mec.pt/pdf/rgci/v12n4/v12n4a11.pdf.

Stori, F., Terra, N. N., Abessa, D. M. S. (2013). Análise das lógicas de ação de atores sociais em torno do licenciamento ambiental de um terminal portuário no estuário de Santos (Brasil). Revista de Gestão Costeira Integrada, 13(3), 365-377. doi: 10.5894/ rgci354.
Vale, M. R. S. (2011). Pesca artesanal na Ilha Diana e meio ambiente: Um estudo de caso. Revista Brasileira de Educação Ambiental (RevBEA), 6(13), 7175. URL: https://periodicos.unifesp.br/index.php/ revbea/article/download/1760/1193/.

Veiga, M. M., Silva, D. M., Veiga, L. B. E., Faria, M. V. C. (2006). Análise da contaminação dos sistemas hídricos por agrotóxicos numa pequena comunidade rural do Sudeste do Brasil. Cad. Saúde Pública, 22(11), 2391-2399. doi: 10.1590/S0102311X2006001100013

Virga, R. H. P., Geraldo, L. P., Santos, F. H. (2007). Avaliação de contaminação por metais pesados em amostras de siris azuis. Ciênc. Tecnol. Aliment., 27(4), 779-785. doi: 10.1590/S0101-20612007000400017 